\title{
Recovering Missing Information in Dislocation-Particle Interactions by Diffraction Contrast Dual-Axes Electron Tomography
}

\author{
G.S. Liu, * I.M. Robertson* \\ * Dept. of Materials Science and Engineering, U. Illinois, 1304 W. Green Street, Urbana IL 61801.
}

Particle strengthening in metallic alloy systems is achieved by a distribution of precipitates in the matrix which serve as barriers to dislocation motion. In order to understand the fundamental processes governing such dislocation-particle interactions, temporal as well as spatially-resolved information on the behavior of dislocations must be known. It has previously been shown that by acquiring a weak-beam dark-field tilt series of an $\mathrm{Al}_{3} \mathrm{Sc}$ particle and the dislocations surrounding it followed by reconstructing the back-projected volume, electron tomography can be used to effectively recover the through-thickness information usually lost in conventional electron micrographs [1]. However, some information remains unrecovered even with this method, most notably some dislocations are excluded from the reconstruction as only one diffraction vector was used to acquire the image; and the "missing wedge" in Fourier space as a result of limited tilt range, of around $+/-40^{\circ}$ on many TEMs. Here we demonstrate how dualaxes electron tomography can be used to capture this missing information to both record the relevant dislocation structure and to mitigate the impact of the missing wedge. The resulting tomogram is then used to construct a three-dimensional model of the volume and this together with the tomogram and $|\mathbf{g} \cdot \mathbf{b}|$ analysis provides complete information and greater insight to dislocation-particle interactions.

Standard $3 \mathrm{~mm}$-diameter discs of a $\mathrm{Cu}-3 \% \mathrm{Co}$ alloy containing semi-coherent precipitates approximately $70 \mathrm{~nm}$ in diameter were prepared and imaged in intervals of $1^{\circ}$ in a double-tilt holder of maximum X-tilt $+/-35^{\circ}$ and $\mathrm{Y}$-tilt $+/-20^{\circ}$. Unlike previous dual-axes tomographic studies, notably Mastronarde et al. [2-4], the tilt-axes for a crystalline specimen are often not orthogonal—in this case, $\mathbf{g}_{1}=\overline{2} 20$ and $\mathbf{g}_{2}=0 \overline{2} 0$. The first and second tilt series were reconstructed to produce individual tomograms, shown in Figure 1a and b, respectively, and then combined to produce the dual-axes tomogram, Figure 1c. Included in the dual-axes tomogram is a correctly oriented Thompson tetrahedron, which can be used to identify slip planes and directions; the procedure for orienting the tetrahedron is described elsewhere [1]. $|\mathbf{g} \bullet \mathbf{b}|$ analysis was performed to obtain possible Burgers vectors of each dislocation. The dual-axes tomogram was used to produce a traced dislocation model, Figure 2, which alleviates issues associated with the limited angular range and number of acquired images. As can be seen in the figures, three of the precipitates have been sheared, and the portion above the slip plane, in yellow, can be distinguished from the original position below the slip plane, in purple. The slip plane was determined to be the $\delta$-plane, but it can be seen from the traced tomogram that the shear direction does not correspond to the slip trace direction - indicating that matrix dislocations with $\mathbf{b}=$ $1 / 2[10 \overline{1}]$ interacted with the particles by shear and subsequently dislocations with $\mathbf{b}=1 / 2[1 \overline{1} 0]$ on the same slip plane glided to produce the slip trace, which intersects the top and bottom surfaces of the sample. The half-loops and individual dislocation segments attached to the interface of the particles are known from dynamic experiments to be associated with room temperature bypass mechanisms leaving behind debris. Several of the half loops reside on two slip planes, indicating cross-slip onto more favorable planes to break away from the particles. 
The observation of shearing of particles of this size and coherency is unexpected and the mechanism is still being investigated. However, the purpose of this paper was to illustrate the use of dual-axes tomography coupled with conventional defect analysis techniques to provide complete three-dimensional information of a defect microstructure, and to introduce the concept of constructing a dislocation model from the tomogram using prior knowledge of dislocations to complete the model. Funding for this work was provided through the Department of Energy [5] and performed at the Center for Microanalysis of Materials, University of Illinois.

\section{References}

[1] G.S. Liu, I.M. Robertson. J. Mater. Res., in press (2011).

[2] D.N. Mastronarde. J. Struct. Bio. 120 (1997) 343-352.

[3] I. Arslan et al. Ultramicroscopy. 106 (2006) 994-1000.

[4] P. Penczek et al. Ultramicroscopy. 60 (1995) 393-410.

[5] This work was supported by the US Department of Energy, Office of Basic Energy Sciences under contract DE-FG02-07ER46443.
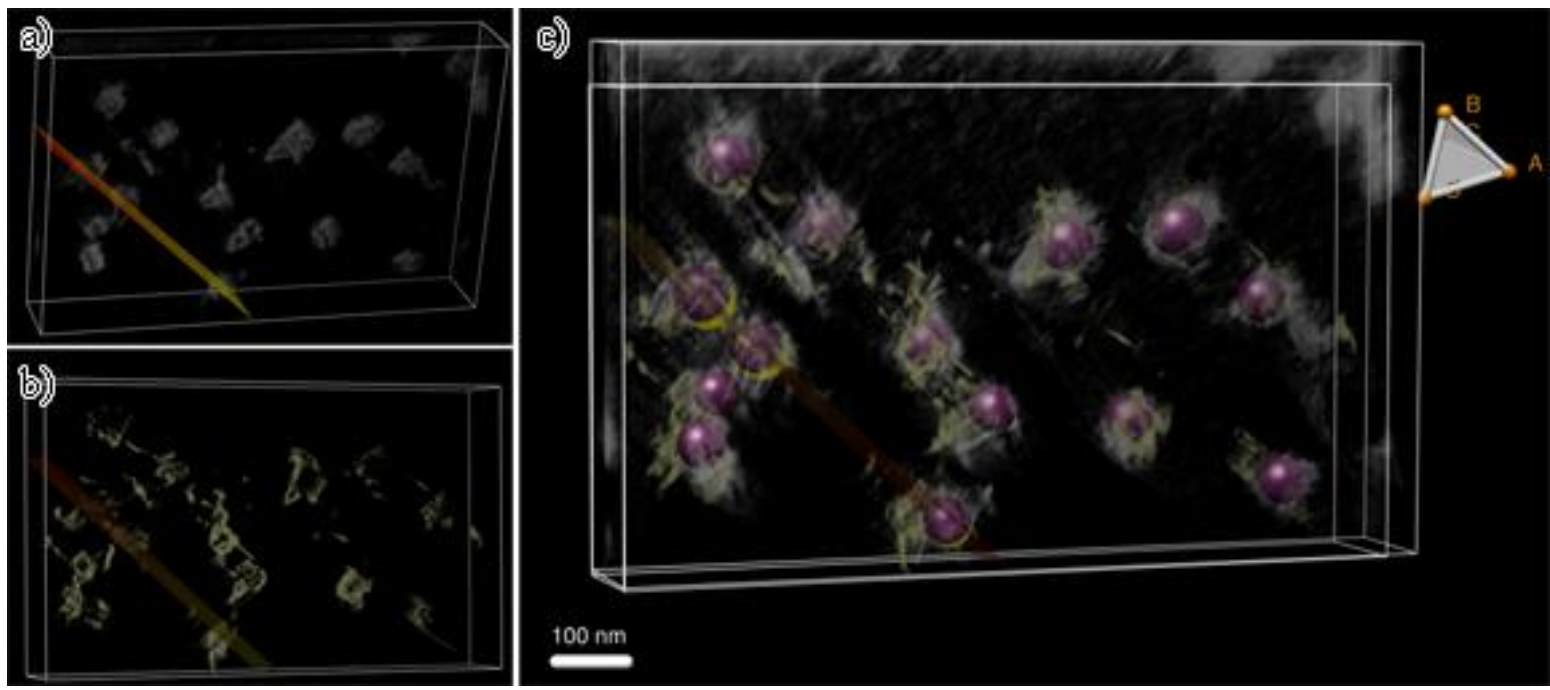

FIG. 1. Tomograms reconstructed from (a) tilt series $1, \mathbf{g}_{1}=\overline{2} 20$, (b) tilt series $2, \mathbf{g}_{2}=0 \overline{2} 0$ and (c) the combined tomogram containing an embedded Thompson tetrahedron and traced particles.


FIG. 2. The traced dislocation model viewed from two different vantage points. (a) From near [11̄2]. The dislocations corresponding to each tilt series are color-coded to show the recovering of dislocations that would otherwise be invisible. (b) From[111]. The particle-shearing direction corresponds to $\mathbf{B C}$ and the slip trace direction to $\mathbf{B A}$. 